BARANKEVICZ GB; NOVELLO D; RESENDE JTV; SCHWARZ K; SANTOS EF. 2015. Características físicas e químicas da polpa de híbridos de tomateiro, durante o armazenamento congelado. Horticultura Brasileira 33: 007-011. DOI - http://dx.doi.org/10.1590/S0102-053620150000100002

\title{
Características físicas e químicas da polpa de híbridos de tomateiro, durante $o$ armazenamento congelado
}

\author{
Gizele B Barankevicz; Daiana Novello; Juliano TV Resende; Kélin Schwarz; Elisvânia F Santos
}

UNICENTRO, Depto. Agronomia, C. Postal 3010,85040-080 Guarapuava-PR; gizele.barankevicz@hotmail.com; nutridai@hotmail. com; jvresende@uol.com.br; kelinschwarz@hotmail.com; elisvania@gmail.com

\section{RESUMO}

O tomate, devido à sua rápida deterioração, torna necessário o congelamento da polpa dos frutos para análises posteriores, porém esse processo pode resultar em perdas consideráveis em sua composição. O objetivo desse trabalho foi avaliar física e quimicamente a polpa congelada de frutos de dois híbridos de tomateiro (Granadero e Tinto). Os frutos foram colhidos no estádio de maturação completo, e em seguida foram triturados. As amostras foram avaliadas antes do congelamento e a cada sete dias, durante o armazenamento a $-18^{\circ} \mathrm{C}$, por 28 dias. As análises foram realizadas em triplicata e avaliou-se os teores de umidade, sólidos solúveis (SS), acidez titulável (AT), ácido ascórbico (AA) e licopeno, assim como a relação sólidos solúveis/acidez titulável (SS/AT) e o pH. Os resultados indicaram que, em geral, os valores de $\mathrm{pH}$ e umidade não apresentaram diferença ( $p>0,05)$ entre os híbridos, bem como ao longo do período de congelamento. Verificou-se que a prática do armazenamento congelado altera a composição da polpa, pois ocorreu diminuição nos teores de SS, AT, AA e aumento nos de licopeno e na SS/AT. O híbrido Granadero apresentou melhores resultados que o Tinto, antes e após o congelamento, mas não se recomenda o armazenamento da polpa destes frutos para análises posteriores.

Palavras-chave: Solanum lycopersicum, ácido ascórbico, compostos bioativos.

\begin{abstract}
Physical and chemical characteristics of tomato hybrids pulp during frozen storage

Tomato requires freezing of pulp for further analyses due to its rapid deterioration. However, this process can result in considerable losses in composition. This study evaluated the physical and chemical features of frozen pulp of two tomato hybrids (Granadero and Tinto). The fruits were harvested at full maturity and crushed, afterwards. Samples were evaluated before freezing and every seven days during storage at $-18^{\circ} \mathrm{C}$ for 28 days. Analyses were carried out in triplicate and moisture, soluble solids (SS), titratable acidity (TA), ascorbic acid (AA) and lycopene were evaluated, as well as the soluble solids/titratable acidity ratio (SS/TA) and $\mathrm{pH}$. Generally, the values of $\mathrm{pH}$ and humidity did not differ $(\mathrm{p}>0.05)$ among the hybrids and throughout the freezing period. Freezing altered the pulp composition, since a decrease in the concentrations of SS, AT, AA and an increase in lycopene and SS/AT was observed throughout the storage period. The hybrid Granadero presented better results than Tinto, before and after freezing, but storage of the pulp of these fruits is not recommended for further analyses.
\end{abstract}

Keywords: Solanum lycopersicum, ascorbic acid, bioactive compounds.

(Recebido para publicação em 7 de março de 2014; aceito em 9 de setembro de 2014)

(Received on March 7, 2014; accepted on September 9, 2014)

$\mathrm{O}$ tomateiro é a segunda hortaliça mais produzida no mundo, sendo uma cultura de grande expressão econômica e social no cenário nacional e internacional, com participação efetiva na dieta humana. $\mathrm{O}$ fruto é considerado fonte de carotenóides, principalmente licopeno e $\beta$ caroteno, das vitaminas B, C e E, de compostos fenólicos como flavonóides, além de conter baixo teor de lipídeos (Ferrari, 2008).

O padrão de qualidade das hortaliças comercializadas no Brasil, geralmente, não é satisfatório, devido à falta de técnicas de pós-colheita adequadas. A qualidade de um fruto, para consumo fresco ou processado, está diretamente relacionada com o estádio de maturação do fruto, pois é ele que define o momento da colheita (Ferreira et al., 2004; Chitarra \& Chitarra, 2005).

A composição nutricional dos alimentos vegetais varia conforme a espécie, estádio de maturação na época da colheita, manuseio pós-colheita e condições de estocagem; a quantidade dos nutrientes nos alimentos in natura pode influenciar a qualidade do alimento processado (Alvarenga, 2004; Correia et al., 2008).

A vitamina $C$ é um fator nutricional especialmente sensível às condições de processamento e sua degradação está relacionada a diversos fatores como temperatura, $\mathrm{pH}$, umidade, presença de substâncias antioxidantes, oxigênio e íons metálicos catalíticos entre outros (Silva et al., 2008). Estudo sobre a cinética de degradação da vitamina $\mathrm{C}$, em condições das funções de processamento, permite escolher processos alternativos ou operações mais eficientes para minimizar as perdas de qualidade (Gabas et al., 2003). Sendo assim, o conhecimento dos principais fatores que afetam a estabilidade das vitaminas torna possível prevenir ou reduzir suas perdas durante a preparação dos alimentos (Moraes et al., 2010).

A quantidade total de carotenóides e, sobretudo, a relação licopeno/beta- 
caroteno é responsável pela coloração final do fruto, variando também em função do amadurecimento (Chitarra \& Chitarra, 2005). Devido a sua estrutura química, o licopeno figura como um dos melhores supressores biológicos de radicais livres, especialmente aqueles derivados do oxigênio. Pesquisas epidemiológicas sugeriram que as propriedades antioxidantes do licopeno são capazes de prevenir diversos tipos de câncer, doenças cardiovasculares e degenerativas (Giovannuci et al., 2002; Carvalho et al., 2005).

Além do fator nutricional, a concentração do licopeno no tomate está relacionada com uma melhor percepção visual dos produtos, existindo, portanto, uma forte demanda para aumentar os teores deste pigmento em frutos das cultivares tanto para consumo in natura quanto para processamento industrial (Boileau et al., 2003). Devido à alta capacidade de oxidação dos carotenóides, o valor nutricional do alimento pode ser reduzido durante as diversas etapas a que são submetidos desde a colheita até a ingestão. Diante disso, é de suma importância prever tais perdas e estabelecer medidas preventivas e critérios que possam ser adotados para minimizar prejuízos em sua composição (Della Lucia et al., 2008).

Geralmente o tomate é consumido fresco e, também, na forma de produtos processados. Porém, muitas vezes, devido a sua rápida deterioração, é necessário o congelamento dos frutos para uma utilização futura, processo que pode resultar em perdas nutricionais consideráveis. Sendo assim, análises físicas e químicas são imprescindíveis para determinar a extensão dessas modificações (Lisiewska \& Kmiecik, 2000).

O objetivo desta pesquisa foi avaliar as características físicas e químicas em amostras congeladas de dois híbridos de tomateiro.

\section{MATERIAL E MÉTODOS}

Obtenção e preparo da matéria -prima - Avaliou-se a polpa de tomates híbridos, Granadero e Tinto, cultivados de forma rasteira, provenientes de experimento conduzido na Universidade
Estadual do Centro-Oeste do Paraná (UNICENTRO), cujo solo é classificado como Latossolo Bruno e o clima subtropical úmido mesotérmico, $\mathrm{Cfb}$, com verões frescos, invernos com ocorrência de geadas severas, e não apresentando estação seca (classificação de Köppen), com temperatura média anual de $18^{\circ} \mathrm{C}$ (Schwarz et al., 2013).

Os frutos foram colhidos no estádio de maturação completa (totalmente amadurecido e desenvolvido), caracterizado pela coloração vermelha descrita por Alvarenga (2004). Somente foram utilizados frutos sadios e com coloração uniforme.

Os tomates foram preparados no laboratório da UNICENTRO. A sanitização dos frutos foi feita lavando-os com água potável, seguida de imersão em solução aquosa contendo $20 \mathrm{mg} / \mathrm{L}$ de agente sanitizante, com concentração de cloro livre de $5 \%$, por 15 minutos (Raupp et al., 2007) e enxágue com água potável.

Para o preparo das amostras, os tomates foram cortados em quatro partes, no sentido longitudinal, e suas sementes removidas. Logo após, foram triturados em liquidificador doméstico (Britania ${ }^{\circledR}$, Brasil) por um minuto em velocidade média. Uma parte da polpa foi utilizada para análise antes do congelamento (tempo zero), e o restante foi embalado em sacos plásticos de polietileno de baixa densidade (PEBD) com capacidade para até $400 \mathrm{~g}$, com espessura de 10 micras, fechados manualmente e armazenados em freezer horizontal, marca Consul ${ }^{\circledR}$ modelo CHB 53C 519L, $\left(-18 \pm 2^{\circ} \mathrm{C}\right)$ para posterior avaliações físicas e químicas.

Análises físicas e químicas da polpa - Obedeceram um delineamento experimental inteiramente casualizado em arranjo fatorial 2x5 (dois híbridos e cinco períodos de armazenamento), totalizando 10 tratamentos, com três repetições. As análises foram realizadas em laboratório da UNICENTRO.

As determinações foram realizadas nas amostras frescas (tempo zero) e congeladas, aos 7, 14, 21 e 28 dias.

a) Sólidos solúveis, acidez titulável e relação sólidos solúveis/acidez titulável

O teor de sólidos solúveis ( $\mathrm{SS}$ ) foi determinado em refratômetro de bancada (marca Optech modelo RMT), sendo os valores expressos em ${ }^{\circ}$ Brix (IAL, 2008).

A acidez titulável (AT) foi determinada por meio de titulação com $\mathrm{NaOH}$ a 0,1 mol/L, até pH 8,2 (IAL, 2008); os resultados foram expressos em $\mathrm{g}$ de ácido cítrico/100 g de polpa. A relação SS/AT foi obtida pela razão entre os teores de sólidos solúveis e de acidez titulável.

\section{b) pH e umidade}

$\mathrm{O} \mathrm{pH}$ foi obtido por leitura com potenciômetro (MS Tecnopon modelo mPA-210), feita diretamente na polpa triturada, sendo seus resultados expressos em unidades de $\mathrm{pH}$, de acordo com AOAC (1995).

O teor de umidade foi determinado por diferença de massa, em amostras submetidas à secagem em estufa com circulação de ar, a $70^{\circ} \mathrm{C}$, até peso constante (IAL, 2008).

\section{c) Licopeno e ácido ascórbico}

O teor de licopeno nos frutos foi obtido por análise espectrofotométrica, com comprimento de onda de $470 \mathrm{~nm}$, segundo o método proposto por Rodriguez-Amaya (2001). O teor de licopeno foi obtido pela seguinte fórmula:

$$
\mu \mathrm{g} \mathrm{g}^{-1}=\frac{(\mathrm{A} \times \mathrm{V} \times 1.000 .000)}{\left(\mathrm{A}_{1 \mathrm{~cm}}^{1 \%} \times \mathrm{M} \times 100\right)}
$$

O teor de ácido ascórbico foi determinado por meio do método titulométrico padrão da Association of Official Analitical Chemists (AOAC, 1984), modificado por Benassi \& Antunes (1988) e expresso em mg de ácido ascórbico/100 g polpa.

Os dados foram submetidos à análise de variância (ANOVA) sendo aplicado o teste de Tukey e $\mathrm{t}$ de student para comparação de médias, ao nível de 5\% de significância. Os resultados também foram submetidos à análise de regressão. Todas as análises foram realizadas com o auxílio do programa SISVAR (Ferreira, 2008).

\section{RESULTADOS E DISCUSSÃO}

O teor de umidade na polpa do tomate Tinto foi maior $(\mathrm{p}<0,05)$ que na 


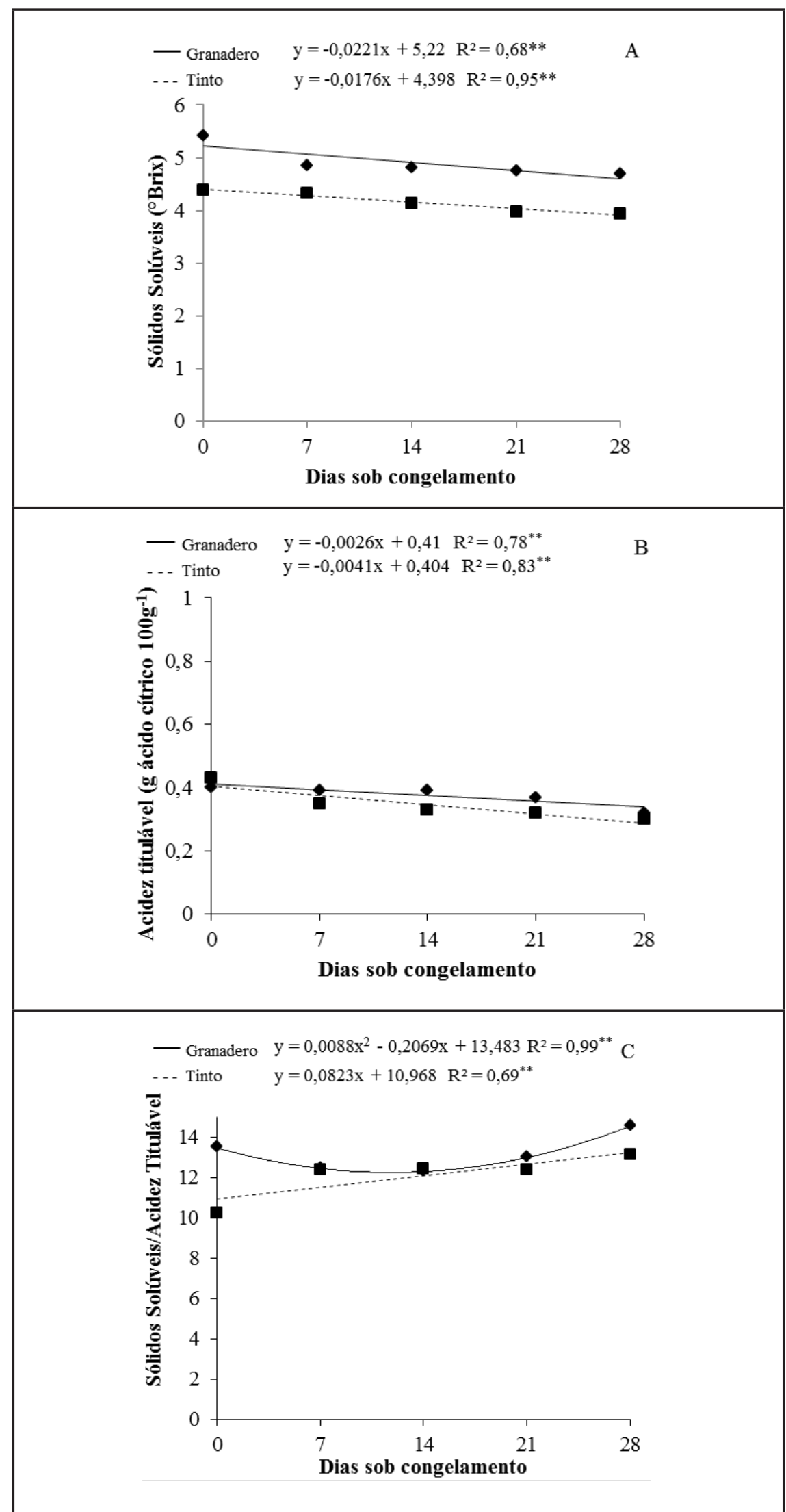

Figura 1. Teor de sólidos solúveis (A), acidez titulável (B) e relação sólidos solúveis/acidez titulável (C) na polpa congelada e armazenada a $-18^{\circ} \mathrm{C}$, de tomates híbridos, Granadero e Tinto; **significativo a $1 \%$ de probabilidade (soluble solids content, titratable acidity and ratio soluble solids/titratable acidity in frozen tomato pulp and stored at $-18^{\circ} \mathrm{C}$ from Granadero and Tinto hybrids; **significant at 1\% probability). Guarapuava, UNICENTRO, 2012. polpa do Granadero (Tabela 1), mas não se observou alteração neste teor durante o período de congelamento em ambos os híbridos, com resultados similares ao da amostra fresca, o que corrobora com os resultados apresentados por Raupp et al. (2007) que encontraram valores variando de 95,5 a 94,4\%, ao avaliarem tomates longa vida antes de serem desidratados. Monteiro et al. (2008) também observaram teores médios de umidade de $95,88 \%$ em tomates frescos sem casca e semente.

$\mathrm{O}$ pH da polpa do híbrido Granadero variou entre 4,03 e 4,08, sendo menor na amostra do híbrido Tinto, em todos os dias de análise, cujos valores variaram de 4,17 a 4,20. Nas amostras do Granadero ocorreu redução no $\mathrm{pH}$, durante o armazenamento congelado $(p<0,05)$. Estes resultados indicam que esta forma de armazenamento não prejudicou este parâmetro que permaneceu dentro da faixa de valores recomendada para 0 consumo, pois valores inferiores a 4,5 são os desejáveis, principalmente para o controle da proliferação de micro-organismos (Monteiro et al., 2008).

O híbrido Granadero apresentou maiores teores $(\mathrm{p}<0,05)$ de sólidos solúveis (SS) que o Tinto, e ambos apresentaram redução nestes teores ao longo do armazenamento congelado (Figura 1A).

Conforme Cheftel (1989), a redução no teor de SS pode ser explicada pelo início das reações catabólicas que conduzem à senescência, o que possivelmente é decorrente do fato de que durante o período de congelamento e mesmo durante o armazenamento a $-18^{\circ} \mathrm{C}$, uma proporção de água ainda está no estado líquido (2-15\%), conservando suas propriedades solvente e reativa, o que não permite a interrupção de algumas reações metabólicas que consomem energia mediante processo respiratório. Segundo Mir \& Beaudry (2002) e Valero \& Altisent (1998), essa redução nos teores de sólidos solúveis pode ser justificada pelo elevado consumo de sólidos para suprir a energia necessária às reações metabólicas.

Durante o período de armazenamento, os teores de acidez titulável (AT) na polpa do híbrido Granadero foram maiores que na do Tinto (Figura 1B), após o $7^{\circ}$ dia de congelamento, e em ambos ela se reduziu, mas com maior 
Tabela 1. Teores médios de umidade e $\mathrm{pH}$ na polpa congelada e armazenada a $-18^{\circ} \mathrm{C}$ de tomates híbridos, Granadeiro e Tinto (content of moisture and $\mathrm{pH}$ in frozen pulp and stored at $-18^{\circ} \mathrm{C}$ Granadero and Tinto hybrids). Guarapuava, UNICENTRO, 2012.

\begin{tabular}{lccccc}
\hline \multirow{2}{*}{ Avaliação } & \multicolumn{5}{c}{ Armazenamento congelado (dias) } \\
\cline { 2 - 6 } & $\mathbf{0}$ & $\mathbf{7}$ & $\mathbf{1 4}$ & $\mathbf{2 1}$ & $\mathbf{2 8}$ \\
\hline Umidade (\%) & & & & & \\
\hline Granadero & $95,00 \pm 0,38 \mathrm{aA}$ & $94,86 \pm 0,41 \mathrm{bA}$ & $94,94 \pm 0,08 \mathrm{bA}$ & $95,08 \pm 0,04 \mathrm{bA}$ & $95,03 \pm 0,02 \mathrm{aA}$ \\
Tinto & $95,42 \pm 0,06 \mathrm{aA}$ & $95,88 \pm 0,07 \mathrm{aA}$ & $95,54 \pm 0,08 \mathrm{aA}$ & $95,59 \pm 0,10 \mathrm{aA}$ & $95,34 \pm 0,25 \mathrm{aA}$ \\
\hline pH & & & & & \\
\hline Granadero & $4,08 \pm 0,01 \mathrm{bA}$ & $4,04 \pm 0,01 \mathrm{bB}$ & $4,03 \pm 0,01 \mathrm{bB}$ & $4,03 \pm 0,01 \mathrm{bB}$ & $4,04 \pm 0,01 \mathrm{bB}$ \\
Tinto & $4,19 \pm 0,01 \mathrm{aA}$ & $4,17 \pm 0,01 \mathrm{aA}$ & $4,20 \pm 0,01 \mathrm{aA}$ & $4,18 \pm 0,01 \mathrm{aA}$ & $4,20 \pm 0,01 \mathrm{aA}$ \\
\hline
\end{tabular}

Médias seguidas pela mesma letra minúscula na coluna (híbridos) e maiúscula na linha (dias) não diferem entre si pelo teste de Tukey $(\mathrm{p}<0,05)$; Os valores que acompanham as médias são o desvio padrão da mesma. (average values followed by same lowercase letter in the column and uppercase letter in the row do not differ statistically, Tukey 5\%).

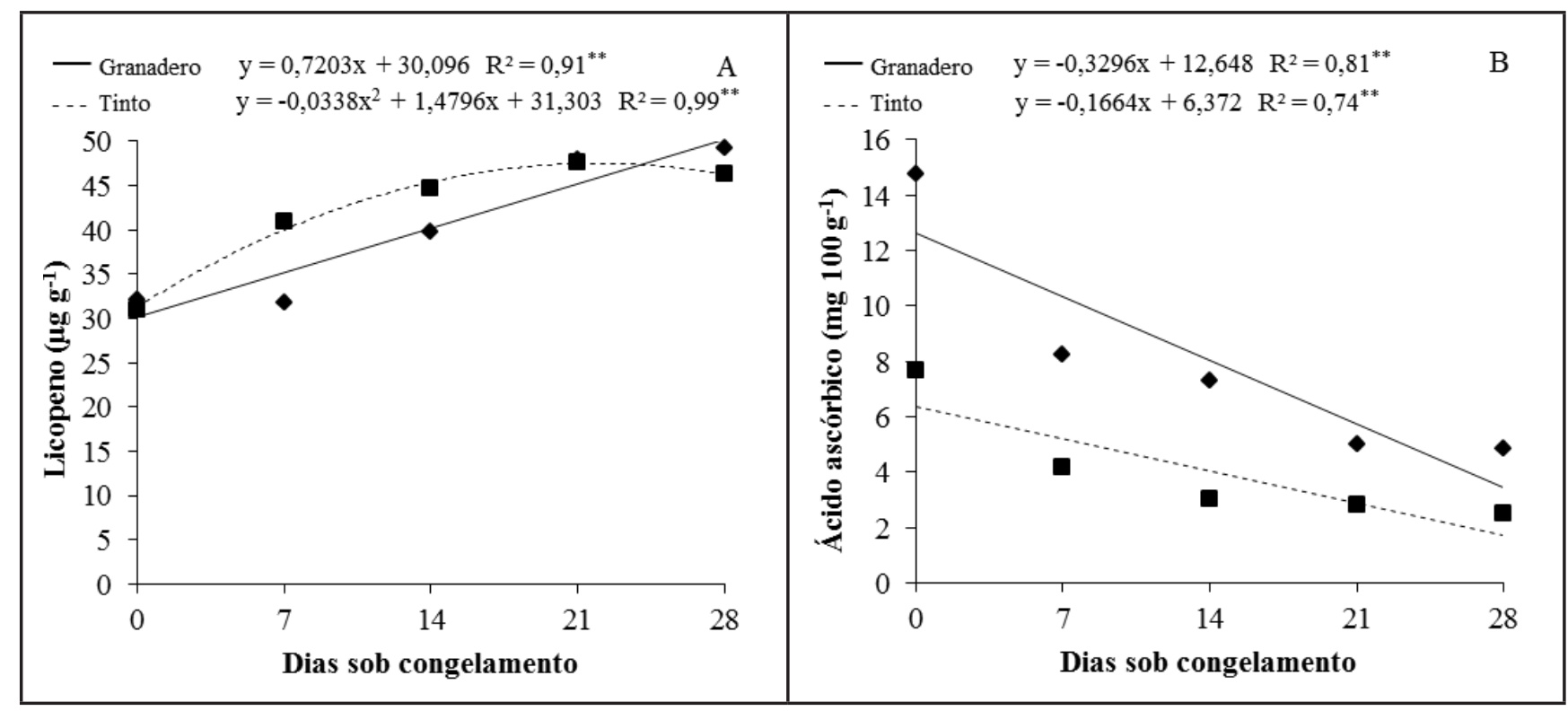

Figura 2. Teor de licopeno (A) e ácido ascórbico (B) na polpa congelada e armazenada a $-18^{\circ} \mathrm{C}$, de tomates híbridos, Granadero e Tinto; ** significativo a $1 \%$ de probabilidade (lycopene content and ascorbic acid content in frozen tomato pulp stored at $-18^{\circ} \mathrm{C}$ from Granadero and Tinto hybrids; **significant at 1\% probability). Guarapuava, UNICENTRO, 2012.

intensidade no Tinto. Esta redução pode ser explicada pelo uso de ácidos como fonte de energia para as reações catabólicas, que não foram totalmente interrompidas pelo congelamento (Melo et al., 2000).

Ressalta-se que os híbridos analisados podem ser considerados produtos com boa qualidade, pois apresentaram mais de $0,32 \%$ de acidez titulável, o que vem ao encontro do indicado por Kader et al. (1978).

A relação SS/AT foi maior na polpa do híbrido Granadero (Figura 1C), que se ajustou de forma significativa ao modelo quadrático, com menor valor aos 11 dias $(12,27)$. Ambos os híbridos apresentaram valores desta relação de acordo com o recomendado por Kader et al. (1978), ou seja, maior que 10, para frutos de alta qualidade. Ressaltase que altos valores para esta relação é indicativo de excelente combinação entre açúcares e ácidos, proporcionando sabor suave ao fruto e melhor aceitação sensorial (Ferreira et al., 2004).

Observou-se variações nos teores de licopeno na polpa dos frutos dos dois híbridos, durante o congelamento, em que o Granadero apresentou aumento linear, enquanto os do Tinto apresentaram aumento até o $22^{\circ} \mathrm{dia}(47,49 \mu \mathrm{g} / \mathrm{g})$, quando se reduziu até o $28^{\circ}$ dia. Segundo Rodriguez-Amaya (2001), em um mesmo alimento diferenças qualitativas e especialmente quantitativas, no teor de carotenoides, ocorrem como resultado de fatores como cultivar e parte da planta amostrada.

Os teores de ácido ascórbico na polpa dos dois híbridos diminuíram $(p<0,05)$ linearmente (Figura 2B), ao longo do período de armazenamento congelado, com quantidades significativamente maiores no Granadero. Presume-se que isto ocorreu devido a oxidação enzimática e não-enzimática do ácido ascórbico na presença de oxigênio, que pode ter ocorrido durante o descongelamento. Resultado similar foi encontrado para morangos congelados convencionalmente, em que o teor de 
ácido ascórbico foi parcialmente degradado (Holzwarth et al., 2012).

Shirahige et al. (2010) analisaram amostras não congeladas e verificaram teores de 21,22 mg/100 g, que são superiores aos encontrados neste trabalho, ou de $14,78 \mathrm{mg} / 100 \mathrm{~g}$ a 7,68 mg/100 g.

Os resultados apresentados indicam que houve redução de $67,18 \%$ no teor de ácido ascórbico na polpa dos dois híbridos, com maior perda a partir do $7^{\circ}$ dia, no Tinto e do $14^{\circ}$ dia, no Granadero. Estes resultados são concordantes com o observado por Lisiewska \& Kmiecik (2000) que relataram uma perda de $71 \%$ do valor inicial no teor de ácido ascórbico em tomates congelados em cubos, após um armazenamento de 12 meses a $-20^{\circ} \mathrm{C}$, e $45 \%$ a $-30^{\circ} \mathrm{C}$.

Dermesonlouoglou et al. (2007) também observaram que o armazenamento por doze meses a $-20^{\circ} \mathrm{C}$, em tomates que não foram pré-tratados com solutos osmóticos alternativos (glicose, oligofrutose e trealose), promoveu a degradação da vitamina C em 56\%, enquanto que os frutos pré-tratados apresentaram uma maior retenção do teor de vitamina $\mathrm{C}$.

Durante o período de armazenamento congelado $\left(-18^{\circ} \mathrm{C}\right)$ da polpa dos híbridos Granadero e Tinto, houve aumento na relação SS/AT e no teor de licopeno e redução na acidez dos frutos, no teor de SS e vitamina $\mathrm{C}$, o que pode comprometer a composição da polpa estudada.

\section{REFERÊNCIAS}

ALVARENGA MAR. 2004 Tomate: Produção em campo, em casa-de-vegetação e em hidroponia. Lavras: UFLA. 400p.

AOAC - Association of Official Analitical Chemists. 1984. Official methods of analisys of AOAC International. Gaithersburg MD, USA, Official method 43.064.

AOAC - Association of Official Analitical Chemists. 1995. Official methods of analysis. $16^{\text {th }}$ ed. Arlington, 45: 18-19.

BENASSI MT; ANTUNES AJ.1988. A comparison of methaphosphoric and oxalic acids as extractant solutions for the determination of vitamin $\mathrm{C}$ in selected vegetables. Brazilian
Archives of Biology and Technology 31: 507-513.

BOILEAU TWM; LIAO Z; KIM S; LEMESHOW S; ERDMAN JUNIOR JW; CLINTON SK. 2003. Prostate carcinogenesis in N-methil-Nnitrosourea (NMU)-testosterone-treated rats fed with tomato powder, lycopene, or energy restricted diets. Journal of the National Cancer Institute 95: 1578-1586.

CARVALHO LA; NETO JT; ARRUDA MC; JACOMINO AP; MELO PCT. 2005. Caracterização físico-química de híbridos de tomate de crescimento indeterminado em função do espaçamento e número de ramos por planta. Revista Brasileira de Agrociência 11: 295-298.

CHEFTEL H. 1989. Introduction a la bioquimicay tecnologia de los alimentos. Zagarosa: Acribia. p.175-299.

CHITARRA MIF; CHITARRA AB. 2005. Póscolheita de frutos e hortaliças: fisiologia e manuseio. Lavras: ESAL. 785p.

CORREIA LFM; FARAONI AS; PINHEIROSANT'ANA HM. 2008. Efeitos do processamento industrial de alimentos sobre a estabilidade de vitaminas. Alimentos $e$ Nutrição 19: 83-95.

DELLA LUCIA CM; CAMPOS FM; MATA GMSC; PINHEIRO-SANT'ANA HM. 2008. Controle de perdas de carotenóides em hortaliças preparadas em unidade de alimentação e nutrição hospitalar. Ciência \& Saúde Coletiva 13: 1627-1636.

D E R M E S O N L O U O G L O U M C ; GIANNAKOUROU MC; TAOUKIS P. 2007. Stability of dehydrofrozen tomatoes pretreated with alternative osmotic solutes. Journal of Food Engineering 78: 272-280.

FERRARI AA. 2008. Caracterização química de tomates (Lycopersicon esculentum) empregando análise por ativação neutrônica instrumental. Piracicaba: USP. $151 \mathrm{p}$. (Dissertação mestrado).

FERREIRA DF. 2008. SISVAR: Sistema de análise de variância versão 5.3. Symposium. Lavras: UFLA. 6: 36-41.

FERREIRA SMR; FREITAS RJS; LAZZARI EN. 2004. Padrão de identidade e qualidade do tomate (Lycopersicon esculentum) de mesa. Ciência Rural 34: 329-335.

GABAS AL; TELIS-ROMERO J; MENEGALLI FC. 2003. Cinética de degradação do ácido ascórbico em ameixas liofilizadas. Ciência e Tecnologia de Alimentos 23: 66-70 (Suplemento).

GIOVANNUCCI E; RIMM EB; LIU Y; STAMPFER MJ; WILLETT WC. 2002. A prospective study of tomato products, lycopene, and prostate cancer risk. Journal of the National Cancer Institute 94: 391-398.

HOLZWARTH S; KORHUMMEL S; CARLE R; KAMMERER DR. 2012. Evaluation of the effects of different freezing and thawing methods on color, polyphenol and ascorbic retention in strawberries (Fragaria $x$ ananassa) Food Research Internacional 48: 241-248.

IAL. INSTITUTO ADOLFO LUTZ. 2008. Brasil. Ministério da Saúde. Agência Nacional de Vigilância Sanitária. Métodos físico-químicos para análise de alimentos. Brasília: Ministério da Saúde. 1020p.

KADER AA; MORRIS LL; STEVENS MA; ALBRIGHT-HOLTON M. 1978. Composition and flavor quality of fresh market tomato as influenced by some postharvest handling procedures. Journal of the American Society for Horticultural Science 103: 6-13.

LISIEWSKA Z; KMIECIK W. 2000. Effect of storage period and temperature on the chemical composition and organoleptic quality of frozen tomato cubes. Food Chemistry 70: 167-173.

MELO EA; LIMA VLAG; NASCIMENTO PP. 2000. Temperatura no armazenamento de Pitanga. Scientia Agricola 57: 629-634.

MIR N; BEAUDRY R. 2002. Atmosphere control using oxygen and carbon dioxide. In: KNEE M (ed). Fruit quality and its biological basis. Columbus: Sheffield Academic 122-149.

MONTEIRO CS; BALBI ME; MIGUEL OG; PENTEADO PTPS; HARACEMIV AMC. 2008. Qualidade nutricional e antioxidante do tomate "tipo italiano". Alimentos e Nutrição 19: 25-31.

MORAES FA; COTA AM; CAMPOS FM; PINHEIRO-SANT'ANA HM. 2010. Perdas de vitamina $\mathrm{C}$ em hortaliças durante o armazenamento, preparo e distribuição em restaurantes. Ciência \& Saúde Coletiva 15: 51-62.

RAUPP DS; GABRIEL LS; VEZZARO AF; DAROS PA; CHRESTANI F; GARDINGO JR; BORSATO AV. 2007. Tomate longa vida desidratado em diferentes temperaturas de secagem. Acta Scientiarum Agronomy 29: 33-39.

RODRIGUEZ-AMAYA DA. 2001. Guide to carotenoids analysis in food. Washington: International Life Sciences Institute Press. 64p.

SCHWARZ K; RESENDE JTV; PRECZENHAK AP; PAULA JT; FARIA MV; DIAS DM. 2013. Desempenho agronômico e qualidade físicoquímica de híbridos de tomateiro em cultivo rasteiro. Horticultura Brasileira 31: 410-418.

SHIRAHIGE FH; MELO AMT; PURQUERIO LFV; CARVALHO CRL; MELO PCT. 2010. Produtividade e qualidade de tomates Santa Cruz e Italiano em função do raleio de frutos. Horticultura Brasileira 28: 292-298.

SILVA MR; SANTOS JÚNIOR RTO; FERREIRA CCC. 2008. Estabilidade da vitamina $\mathrm{C}$ em cagaita in natura e durante a estocagem da polpa e refresco. Pesquisa Agropecuária Tropical 38: 52-59.

VALERO C; ALTISENT MR. 1998. Equipos de medida de calidad organoléptica en frutas. Fruticultura Profesional 95: 38-45. 